\title{
DNA damage response and hematological malignancy
}

\author{
Masatoshi Takagi ${ }^{1}$ (1)
}

Received: 28 February 2017 / Revised: 27 March 2017 / Accepted: 28 March 2017 / Published online: 3 April 2017

(C) The Japanese Society of Hematology 2017

\begin{abstract}
DNA damage is a serious threat to cellular homeostasis. Damaged DNA leads to genomic instability, mutation, senescence, and/or cell death. DNA damage triggers a cellular response called the DNA damage response (DDR), followed by activation of the DNA repair machinery. DDR both maintains cellular homeostasis and prevents cancer development. Germ line mutation of DDRassociated genes can lead to cancer-susceptible syndromes. Somatic mutation of DDR-associated genes has also been reported in various tumors, including hematological malignancies. Therapeutic approaches that target the DDR and DNA repair are thus now being developed. Understanding the mechanism(s) underlying DDR and DNA repair will increase our knowledge of cancer etiology and facilitate development of cancer therapies.
\end{abstract}

Keywords DNA damage response - DNA repair - ATM · Hematological malignancy $\cdot$ Caner susceptible syndrome

\section{Concept of DNA damage response}

Cells constantly suffer various stresses (Fig. 1). DNA damage is one of the cellular stresses. DNA damages are artificially induced by exposure to irradiation or anti-tumor agents. Not only by these exogenous factors, but also human cells naturally suffer DNA damage from cellular metabolic or hydrolytic processes or DNA replication

Masatoshi Takagi

m.takagi.ped@tmd.ac.jp

1 Department of Pediatrics and Developmental Biology, Tokyo Medical and Dental University (TMDU), Yushima 1-5-45, Bunkyo-ku, Tokyo 113-8519, Japan errors. The metabolites, reactive oxygen species, reactive nitrogen species, reactive carbonyl species, lipid peroxidation products affect DNA integrity. Other than these metabolites, V(D)J recombination process mediated by RAG1/2 during lymphocyte development involves DNA double-strand breaks (DSB). DNA topoisomerase 2 also induces DSB to control DNA topologic status. Deamination induced by deaminases or depurination leads to DNA single-strand breaks (SSB). SSBs can also arise by DNA topoisomerase 1. Tens of thousands of SSBs, 10-50 DSBs per cell per day occur naturally [1-3].

\section{ATM is a key regulator of DNA damage check point}

Once cells suffer DNA damage, cells try to resolve damages. The cellular response to DNA damage induces cell cycle arrest, damaged DNA repair, or apoptosis. This machinery is called DNA damage response (DDR), or DNA damage checkpoint. Numerous factors are involved in this process. The central player in DNA damage response, especially in DSB, is ATM. DNA damage response comprised several steps, sensor, transducer, and effector. When DNA DSB happen, the sensor, MRE11RAD50-NBN (MRN) complex, is recruited to the DSB site [4]. ATM is present as a dimer or higher order multimer in unstressed cell with the kinase domain bound to a region surrounding Ser 1981 that is contained within the FAT domain. Cellular irradiation induces rapid intermolecular autophosphorylation of serine-1981 and this phosphorylation event results in dimer dissociation and initiation of cellular ATM kinase activity [5]. Then activated ATM recruited DSB site. Activated ATM transduces the signal to downstream effectors by phosphorylating 

damage and types of DNA damages. Various exogenous and endogenous factors that induce DNA damage
Fig. 1 Factors that induce DNA
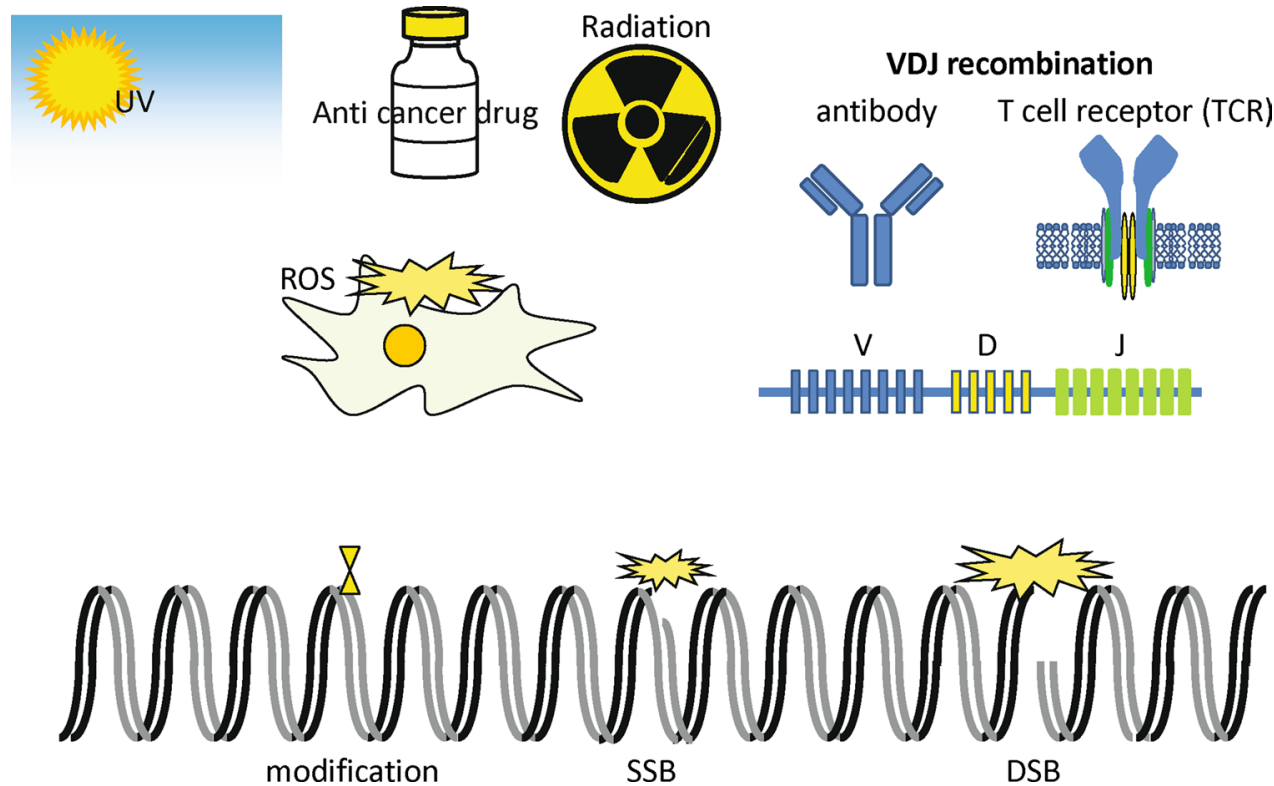

substrates. Huge number of ATM substrates is reported [6]. Most of them work as effector for cell cycle arrest, DNA repair, apoptosis, gene expression or cellular homeostasis.

\section{ATM and cell cycle check point}

\section{G1/S check point}

Importance of ATM is particularly exhibited in cell cycle regulation after DNA damage. Cells that suffered DNA damage should stop the progression of cell cycle and determine whether damage is repairable or not. If it is repairable, cells stop in the cell cycle phase and activate repair machinery. If the damage is catastrophic for cells, cell decides activation of cell death signaling pathway including apoptosis. ATM-TP53 pathway plays a critical role for G1/S arrest after DNA damage. TP53 induction after irradiation is dependent on ATM under fine-tuning of phosphorylation and inhibiting degradation by MDM2 [7]. TP53 Ser 15 residue is phosphorylated by ATM directly, and Ser 20 is phosphorylated by CHEK2, activated by ATM-dependent Thr 68 phosphorylation [8, 9]. MDM2 Ser 394 is also phosphorylated by ATM. Phosphorylated MDM2 may be less capable of promoting the nucleo-cytoplasmic shuttling of TP53 and its subsequent degradation, thereby enabling TP53 accumulation [10]. Accumulation of TP53 subsequently induces CDKN1A (p21) transcriptional expression. Then, CDKN1A (p21) inhibits CCNE (cyclin E)/CDK2, CCND1 (cyclin D1)/ CDK4 or CDK6 leading to cell cycle arrest at G1/S boundary.
Intra-S phase check point

A $\mathrm{T}$ cell is known to exhibit radiation-resistant DNA synthesis (RDS). The precise mechanism of RDS is not fully understood. But several key players associated with intra$S$ phase check point are reported. CHEK2-dependent CDC25A phosphorylation is one of the main regulators in $S$ phase progression [11]. To prevent DSBs during replication, stalled forks need to restart before collapsing. Homologous recombination repair (HRR) can escort stalled forks to restart before collapsing. Therefore, molecules involving HRR is regulated by ATM/ATR-dependent phosphorylation after DNA damage. Various ATM substrates including and BRCA1 are also cooperatively working at intra-S phase check point [12].

\section{G2/M check point}

Activation of CCNB/CDK1 induces mitosis. Phosphorylation of CDK1 at residues Thr14 and Tyr15 by WEE1 and MYT1 inactivates CCNB/CDK1 activity. To enter mitosis, CDK1 is dephosphorylated by CDC25C. PLK1 activated by AURKA (Aurora A) and BORA, subsequently activates $\mathrm{CDC} 25 \mathrm{C}$ and inactivates WEE1 and MYT1. After DNA damage in G2, ATM/ATR-dependent CHEK1 and CHEK2 phosphorylate CDC25C, and inactivate. ATM/ATR directly phosphorylates BORA at Thr 501 residue. Phosphorylated BORA is degraded by E3 ubiquitin ligase SCFBTRC. Once BORA is destructed, PLK1 cannot be activated [13]. As a result, CCNB/CDK1 activity is inhibited. Although ATM and CHEK2 control the establishment of an arrest after damage, ATR and CHEK1 work for checkpoint M/R/N complex, MDC1, TP53BP1, FANCD2, SMC1A 
maintenance in G2. As has been observed in intra-S phase check point, homologous recombination repair by PALB2and BRCA2-dependent strand invasion also plays an important role in $\mathrm{G} 2$ check point [14].

\section{Mitotic spindle assemble check point}

During mitosis, spindle assemble check point prevents anaphase onset until the duplicated chromosome is properly separated, aligned and attached to the spindle.

The spindle assemble check point stops the cell cycle by negatively regulating $\mathrm{CDC} 20$, thereby preventing the activation of the polyubiquitination activities of anaphasepromoting complex (APC). The proteins responsible for the spindle assembly check point signal compose the mitotic check point complex including, MAD1, MAD2, BUB1, CDC20, AURKB, etc. $[15,16]$.

ATM is activated in mitosis in the absence of DNA damage by phosphorylation on serine 1403 by AURKB (Aurora B). Activated ATM phosphorylates BUB1, a critical kinetochore protein, on Ser 314 [17]. ATM-mediated BUB1 phosphorylation is required for BUB1 activity and is essential for the activation of the spindle checkpoint. MAD1 Ser 214 is also phosphorylated by ATM, and contributes to activation of the spindle assembly check point and the maintenance of chromosomal stability (Fig. 2).

\section{DNA repair}

Once DNA damage occurs, cells mobilize various DNA repair machineries. Type of damaged DNA determines appropriate DNA repair machinery. Mainly, DNA repair pathway is divided into two types based on damaged type, single-strand DNA damage and double-strand DNA breaks.

\section{Base and single-strand DNA damage}

When DNA base is damage, damaged DNA is repaired by excision repair (Fig. 4). There are two types of excision repair, base excision repair (BER) and nucleotide excision repair (NER). BER removes small, non-helix-distorting base lesions from the genome. On the other hand, NER repairs bulky helix-distorting lesions. Two types of BER, shortpatch (where a single nucleotide is replaced) or long-patch BER (where 2-10 new nucleotides are synthesized) are used properly based on the damaged lengths (Fig. 3). NER is particularly important for repairing pyrimidine dimer induced by ultraviolet light (UV). By the type of damage recognition, there are two types of NER, global genomic NER (GGR) and transcription-coupled NER (TCR). GGR and TCR share same process for lesion incision, repair, and ligation. DNA mismatch repair (MMR) repairs insertion,
Fig. 2 DNA double-strand breaks and ATM-dependent DNA damage response. Cell cycle regulation by DDR is shown. M/R/N: MRE11A/ RAD50/NBN complex; Gene symbol/Alias combination: CCNE1/Cyclin E, CCNEA2/ Cyclin A2, CCNB/Cyclin B, CDKN1A/p21, H2AFX/H2AX, DCLREC1C/ARTEMIS

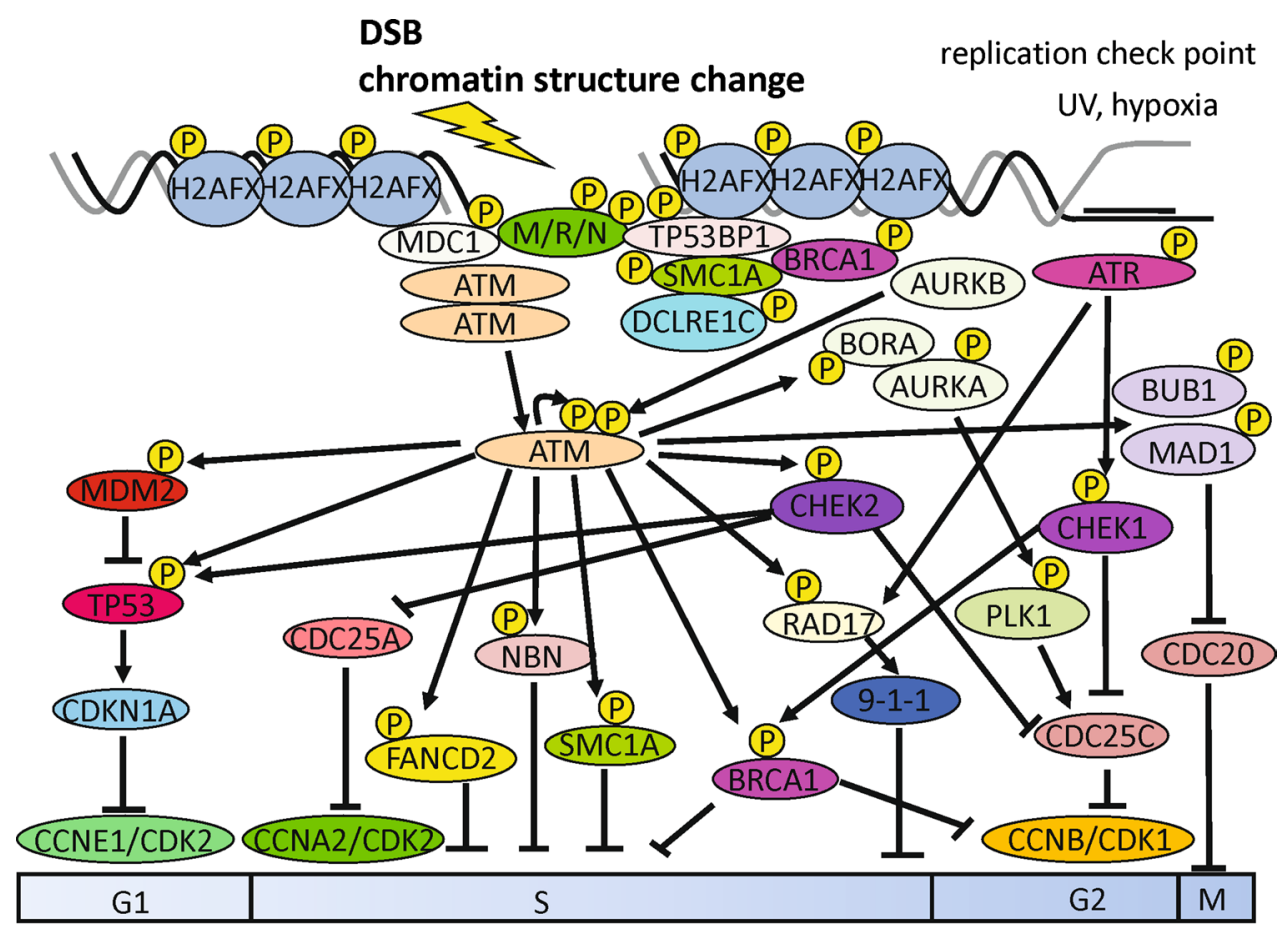

M/R/N; MRE11A/RAD50/NBN complex, Gene symbol (Alias): CCNE1 (Cyclin E), CCNEA2 (Cyclin A2), CCNB (Cyclin B), CDKN1A (p21), H2AFX (H2AX), DCLRE1C (ARTEMIS). 

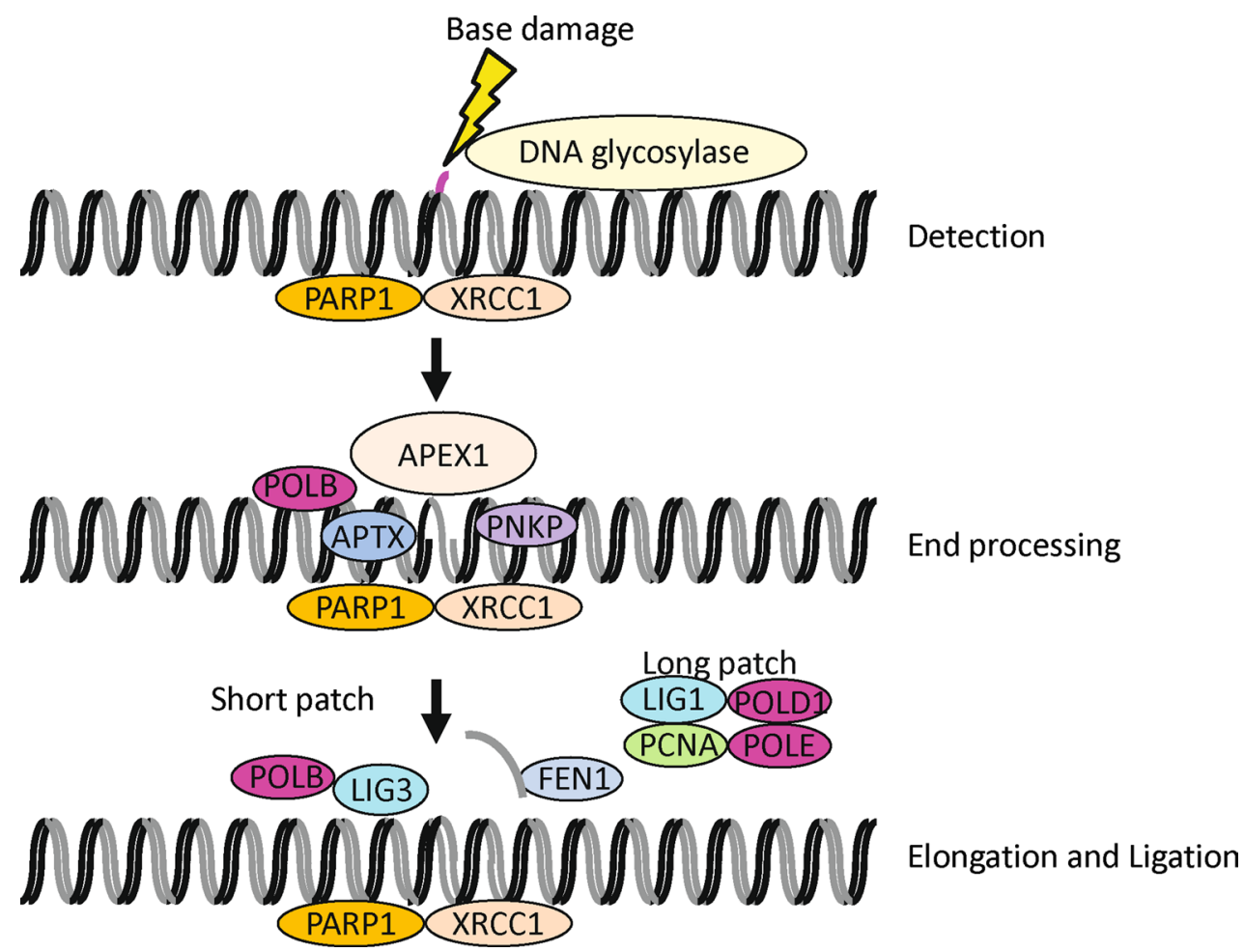

Fig. 3 Base excision repair. Recognition and removal of an incorrect or damaged substrate base by a DNA glycosylase to flip out damaged base, leaving an abasic site (AP-site). AP site incision by an apurinic/apyrimidinic (AP) endonuclease, APEX1. As 5'- or 3'-blocking ends are refractory to DNA polymerase repair synthesis or nick ligation. POLB can remove the blocking moiety to create the required $5^{\prime}-\mathrm{PO}_{4}$ for DNA ligase nick sealing. PNKP is the primary enzyme for removing $3^{\prime}-\mathrm{PO}_{4}$ blocking groups. APTX is able to remove the

deletion, and mis-incorporation of bases during DNA replication and recombination. Defect of MMR increases the spontaneous mutation rate. MMR system is required for cell cycle arrest and/or programmed cell death in response to certain types of DNA damage. MSH2 and MLH1, molecules involving in MMR, interact physically with ATM, ATR-ARTIP in cells treated with DNA-damaging agents. This observation links MMR and DDR. Inactivation of MMR in human cells is associated with hereditary and sporadic human cancers. Biallelic mutation of MMR-associated genes develops constitutional mismatch repair deficiency (CMMRD) syndrome-susceptible solid tumor as well as hematological malignancy (see later chapter).

\section{DNA double-strand breaks}

Non-homologous end joining (NHEJ)

NHEJ is an error-prone repair pathway (Fig. 4). Not only DSBs generated by external factors but also V(D)J 5'-adenylated-deoxyribose phosphate (5'-AMP-dRP) group. Then, gap is filled by a POLB for short-patch BER. FEN1 remove the displaced strand created by POLB, POLE, POLD1 and PCNA for longpatch BER. Nick is sealed by a LIG3 for short-patch BER and a LIG1 for long-patch BER. PARP1 and XRCC1 are needed to stabilize DNA strand breaks until repair can take place to prepare ends for gap-filling synthesis and nick ligation

recombination utilizes NHEJ. DSB is directly rejoined by NHEJ. The factors associated with NHEJ are responsive genes for sever combined immunodeficiencies (SCID). LIG4 syndrome is caused by mutation of LIG4. SCID caused by mutation of DCLRE1C (ARTEMIS) is one of representative diseases caused by defect of NHEJ. Major cancer susceptibility is not known by defect of molecules involving NHEJ.

\section{Homologous recombination repair (HRR)}

Homologous recombination repair is an error-free repair pathway (Fig. 5). HRR uses newly synthesized (daughter) strand for recombination repair. Therefore, HRR occurs during $\mathrm{S}$ to $\mathrm{G} 2$ phase. The factors associated with HRR are responsive genes for several cancer susceptibility syndromes. BRCA1/2, the responsible gene for hereditary breast and ovarian cancer, is one of representative genes involving HRR. 
Fig. 4 Non-homologous end joining. MRN complex is recruited to DSBs early and is thought to promote bridging of the DNA ends. The initial step in NHEJ is the recognition and binding of the KU70/80 heterodimer to the DSB. Then, KU70/80 heterodimer recruits PRKDC (DNA-PK), XRCC4, NHEJ1 (XLF/CERENUNNOS), and LIG4. PRKDC can phosphorylate several own stream targets. NHEJ requires two DNA blunt ends to join them together. Single-strand overhangs are processed by DCLRE1C (ARTEMIS). After creation of blunt ends, XRCC4/ LIG4 complex mediates ligation of the DSB
Interstrand crosslink (ICL)

Interstrand crosslinks are highly toxic DNA lesions that prevent transcription and replication by inhibiting DNA

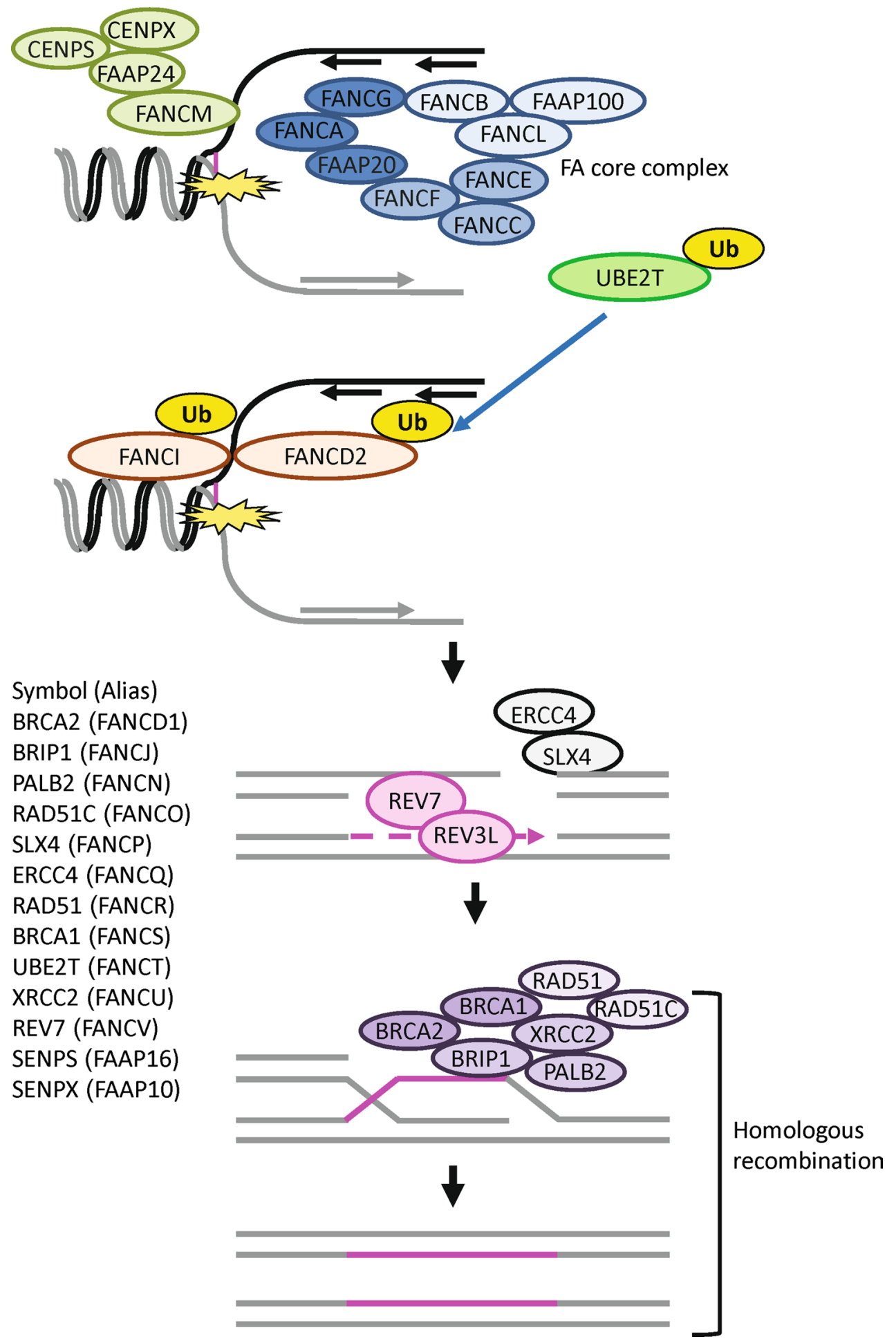

strand separation. If ICLs are not repaired properly, ICLs are converted to DSB during DNA replication. FanconiBRCA1 pathway is one of a main pathway works in ICLs (Fig. 6). 
Fig. 5 Homologous recombination repair. Homologous recombination is initiated by the binding of the MRN complex and ATM. The nuclease-bearing subunit MRE11A and RBB8 (CTIP) initiates the nucleolytic processing of the $5^{\prime}$ ends at the DNA break site (which is known as $5^{\prime}-3^{\prime}$ resection). Created single-strand DNA is rapidly bound by the RPA. The RPA is replaced with RAD51, which is mediated by BRCA1, PALB2 and BRCA2. Then, homology search and DNA strand invasion are mediated by the RAD51. DNA strand exchange between the target DNA and the Rad51 filament leads to a structure known as the displacement loop (D-loop). After strand invasion, a DNA polymerase extends the end of the invading $3^{\prime}$ strand.

This changes the D-loop to a cross-shaped structure known as a Holliday junction. Three different pathways, synthesisdependent strand annealing (SDSA), break-induced replication (BIR) and double-strand break repair (DSBR), resolving overhang are proposed

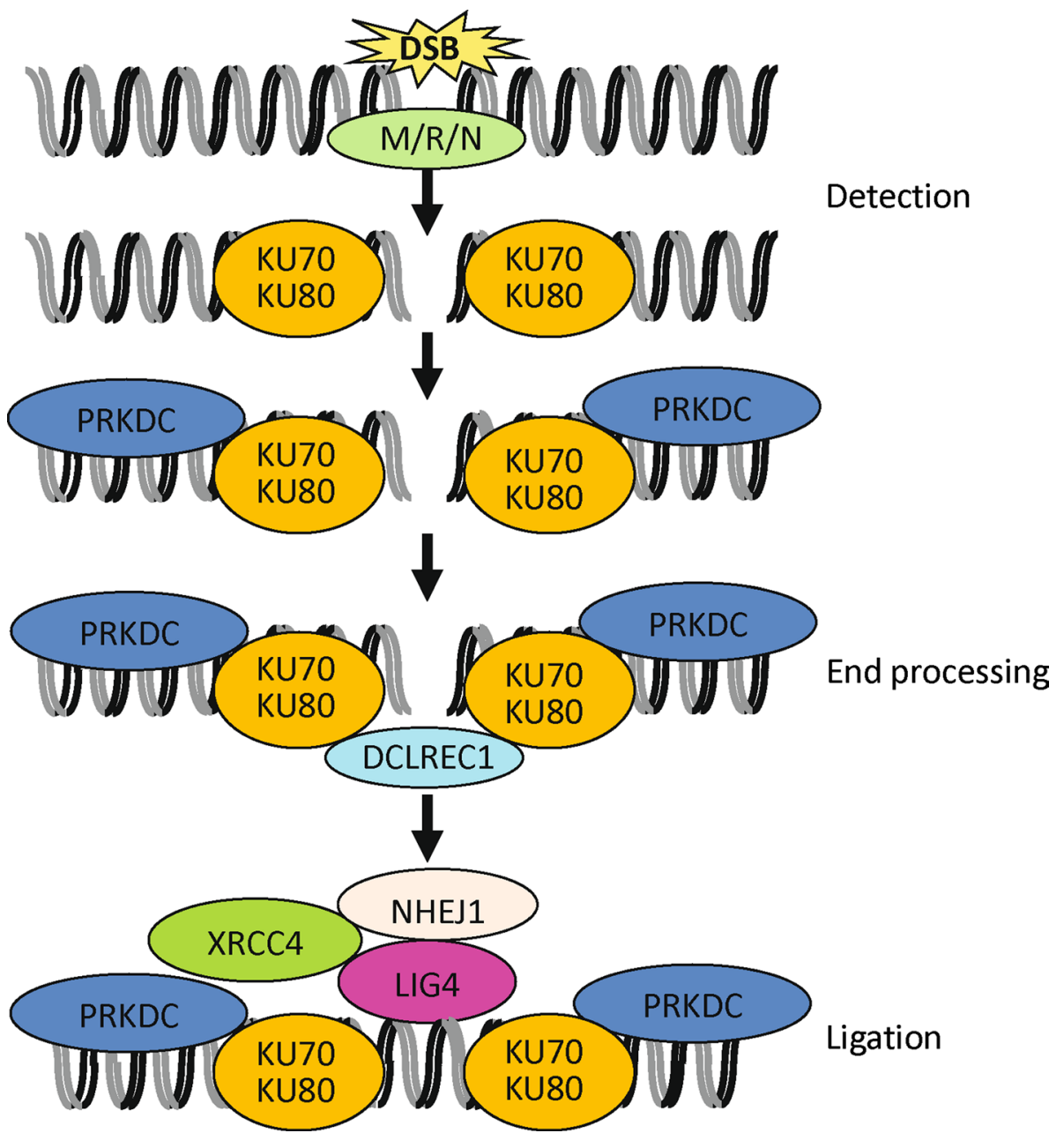

\section{Germ line mutation of DNA damage response-associated gene in hematological ataxia- telangiectasia}

A- $\mathrm{T}$ is a progressive neurodegenerative disorder with onset of truncal ataxia usually before 3 years of age. Ocular apraxia is also present in most of these patients. By age 10, they are wheelchair-bound because of ataxia. One-third of patients have severe immunodeficiency, accompanied by severe sino-pulmonary infections with non-opportunistic organisms. Conjunctival telangiectasia is a well-known characteristic feature which appears several years after the beginning of neurologic symptoms [18].

Serum alpha-fetoprotein (AFP), chromosomal translocations involving chromosomes 7 and 14, and a decrease in the lymphocytes of B cell and $\mathrm{T}$ cell compartments are the most useful tests for the diagnosis of A-T. AFP is elevated in almost all the cases of A-T and is the most useful clinical laboratory test for the diagnosis on the bed side. $\mathrm{T}$ cell numbers are usually low, and many patients show only marginal deficiencies. $\gamma / \delta$ T cell numbers are usually elevated. B cell numbers are normal or slightly elevated. Deficiencies of IgE, IgG2, and IgA are often marked. Glucose intolerance is also seen frequently, allowing the speculation that ATM may somehow be involved in the pathogenesis of DM in the general population.

Radiosensitivity is the most traditional and reliable test to confirm the clinical diagnosis of A-T. Colony survival assay and radioresistant DNA synthesis have been validated for clinical diagnostic testing and colony survival assay was reported to be one of the most reliable assays to identify A-T patients [19].

The responsible gene in A-T is localized at chromosome $11 \mathrm{q} 22.3$ and is called the ATM gene, which contains 66 exons spanning approximately $150 \mathrm{~kb}$ of genomic DNA. Its cDNA consists of $10140 \mathrm{bp}$. ATM mutation in $\mathrm{A}-\mathrm{T}$ is inherited in an autosomal recessive manner. The frequency of A-T patients is estimated as one in 40,000 to 300,000 , and heterozygous carriers of the ATM gene mutation reportedly comprise $0.5-1 \%$ of the general populations. 
Fig. 6 Fanconi pathway. Following exposure to DNAdamaging agents the FANCM anchor complex, comprising FANCM, FAAP24, CENPS and CENPX, recognizes the damage, remodels the fork, and promotes the recruitment of the FA core complex. The FA core complex, which is comprised of three sub-complexes-FANCB/ FANCL/FAAP100, FANCC/ FANCE/FANCF, and FANCA/ FANCG/FAAP20 - together with the E2 ubiquitin-conjugating enzyme UBE2T/FANCT, constitutes an active multisubunit E2/E3 ubiquitination enzyme complex. This E2/E3 enzyme complex catalyzes the site-specific monoubiquitination of FANCD2 K561 and FANCI K523. Schematic of downstream steps of the FA pathway. During the later stages of ICL repair, SLX4 and ERCC4 catalyze the unhooking of the ICL. The unhooked ICL is then bypassed by REV7 and REV3L. Downstream of FANCD2 and FANCI monoubiquitination, HRR facilitates DNA repair [30]
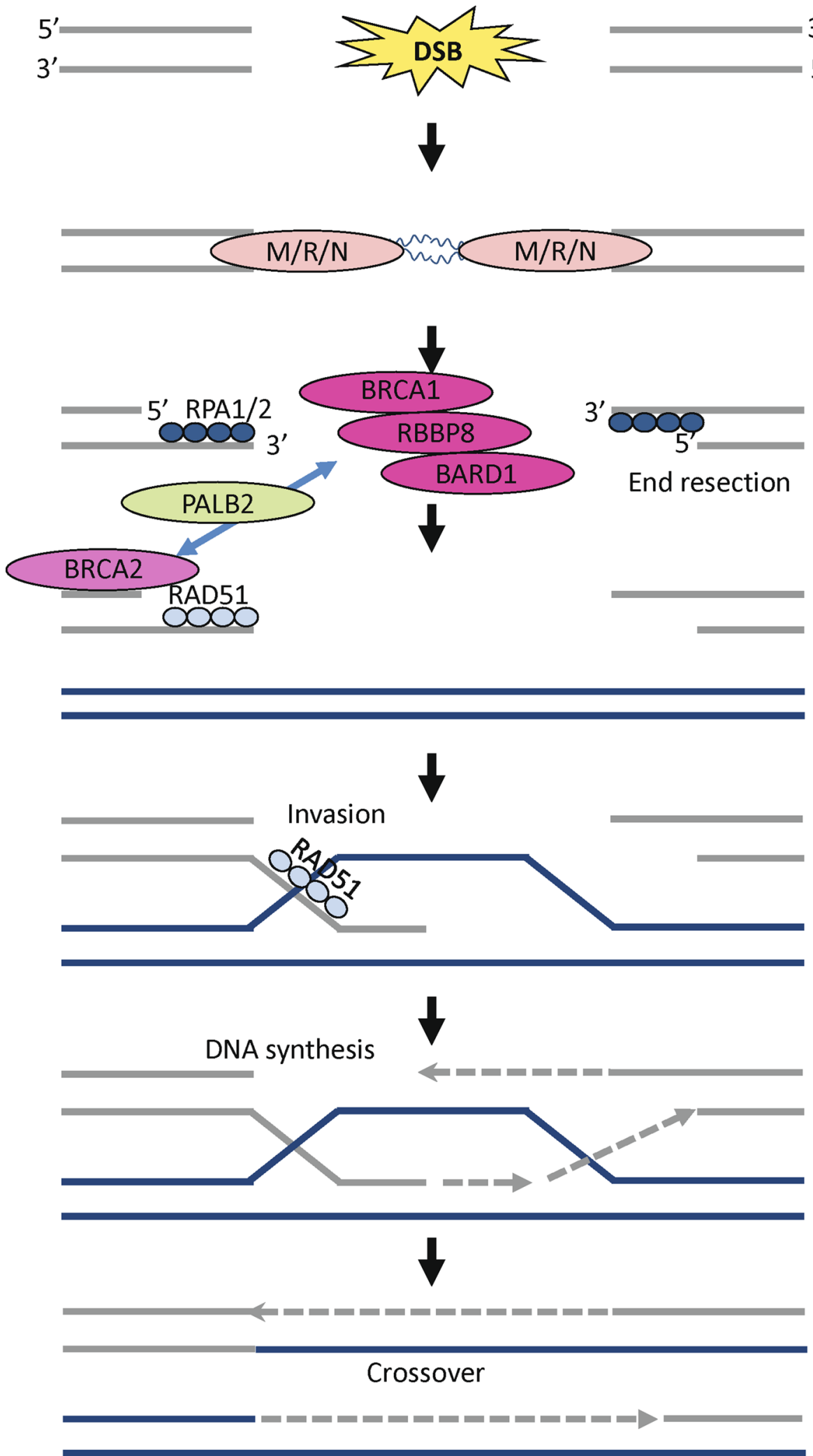


\section{Nijmegen breakage syndrome (NBS)}

The Nijmegen breakage syndrome is an autosomal recessive chromosomal instability syndrome characterized by microcephaly, microgenia, "bird-like" face, growth retardation, mild mental retardation, immunodeficiency, and a predisposition to cancer with normal serum levels of alpha-fetoprotein. Cancer susceptibility is much greater than A-T. However, patients with NBS have neither ataxia nor telangiectasia. Almost all patients reportedly originate from East Europe, and are homozygous for one founder hypomorphic NBN (NBS1) mutation c.657_661del5 originated from Slavic lines. Cells from NBS patients exhibit similar features with those of A-T, namely hyper-radiosensitivity, radioresistant DNA synthesis and chromosomal rearrangement involving TCR and Ig loci [20].

\section{Li-Fraumeni syndrome (LFS)}

The most common mutation in cancer occurs in the TP53 gene. TP53 serves as a tumor suppressor by regulating the expression of cell cycle-, apoptosis-, and metabolism-associated genes such as MDM2, CDKN1A, $\mathrm{BAX}$ and $\mathrm{BBC} 3$ via binding to TP53 DNA recognition sequences located adjacent to the genes. Expression of TP53 in response to DNA damage leads to either cell cycle arrest or apoptosis [21, 22]. Li-Fraumeni syndrome (LFS) is a cancer-prone syndrome. Germ line mutations of TP53 are observed in approximately $75 \%$ of families with LFS and in $40 \%$ of families with $\mathrm{Li}-$ Fraumeni-like syndrome (LFL) [23]. The majority of TP53 mutations identified in LFS patients are missense mutations that alter the structure of the DNA-binding region, and these mutations function as dominant negative mutations by a gain-of-function mechanism.

The tumors most closely associated with LFS are: soft tissue sarcoma, osteosarcoma, pre-menopausal breast cancer, brain tumors, and adrenocortical carcinoma. Hematological malignancy in LFS is less frequent than sarcoma $[24,25]$. On the other hand, the TP53 alterations observed in $91.2 \%$ of childhood low-hypodiploid ALL are present in non-tumor cells in $43.3 \%$ of mutated cases, suggesting that these mutations are inherited and that low-hypodiploid ALL represents a manifestation of Li-Fraumeni syndrome Intriguingly, the TP53 mutations identified in low-hypodiploid ALL included missense, nonsense and insertion/deletion mutations and are in general predicted to be loss-of-function mutations [26].

\section{Bloom's syndrome (BS)}

Bloom's syndrome (BS) is characterized by severe pre- and postnatal growth deficiency, reduced subcutaneous fat tissue, an erythematous, sun-sensitive skin, and immunodeficiency. Cancer susceptibility is observed in BS. More than one-third of patients develop malignant neoplasm at the mean age at diagnosis being 24.8 years [27]. Epithelial cancer and hematological malignancy including lymphoid lineage and myeloid lineage are common. Increased frequency of sister chromatid exchange (SCE)s is demonstrable in BS cultured cells. The gene mutated in BS is The RecQ gene family, BLM. BLM exerts its function as a DNA helicase, and plays an important role in DNA replication and homologous recombination [28].

\section{Fanconi anemia (FA)}

Fanconi anemia (FA) is a rare autosomal and X-linked genetic disease characterized by progressive bone marrow failure (BMF), congenital abnormalities, short stature, abnormal skin pigmentation, skeletal malformations of the upper and lower limbs, microcephaly, and ophthalmic and genitourinary tract anomalies, and increased cancer risk during early adulthood. The incidence of acute myeloid leukemia is $13 \%$. Not only leukemia, solid tumor is also observed in FA patients [29].

The proteins encoded by the FA genes function together in the FA-BRCA pathway to repair DNA damage and to maintain genome stability. Twenty-one disease-causing genes have been identified [30]. An estimated incidence of 1 in 360,000 live births and a carrier frequency of approximately 1 in 181 are observed. One major function of the FA-BRCA pathway is to orchestrate the repair of DNA interstrand crosslinks.

\section{Constitutional mismatch repair deficiency (CMMRD) syndrome}

Heterozygous monoallelic germ line loss-of-function mutations in one of the four MMR genes (MLH1, MSH2, MSH6 or PMS2) are responsible for Lynch syndrome, which develops colorectal cancer, endometrial carcinoma and other malignancies in the fourth and fifth decades of life [31]. Rare individuals carry biallelic homozygous or compound heterozygous deleterious germ line mutations in MMR genes leading to the recessive 
constitutional mismatch repair deficiency (CMMRD) syndrome. CMMRD follows an autosomal recessive inheritance trait. CMMRD patients develop various types of cancers in infancy to adolescence [32]. CMMRD patients exhibit cutaneous phenotype commonly described as an association of café-au-lait macules (CALMs) and other features reminiscent of neurofibromatosis type 1 (NF1). The tumor spectrum of CMMRD is characterized by childhood brain tumors and hematological malignancies and gastrointestinal cancer.

The median age at diagnosis of hematological malignancies and brain tumors was, respectively, 6.6 (1.2-30.8) and 10.3 (3.3-40) years, whereas gastrointestinal cancer occurred later (median age at diagnosis: 21.4 years (11.436.6) [33].

\section{Somatic mutation of DNA damage response-associated gene in hematological malignancies}

\section{ATM mutation and hematological malignancy}

T cell prolymphocytic leukemia (T-PLL), a rare malignancy with similarities to a mature $\mathrm{T}$ cell leukemia is frequently observed in ataxia-telangiectasia. High frequency of ATM mutations in T-PLL-developed non-A-T patient was identified [34]. Most of A-T is caused by truncation mutation of ATM. However, mutations in T-PLL were missense mutations. Based on this observation, ATM mutation was investigated in a variety of lymphoid malignancies, and identified in B-chronic lymphocytic leukemia (CLL), B cell non-Hodgkin lymphomas (B-NHL) and Mantle cell lymphoma (MCL).

$20-30 \%$ of B cell chronic lymphocytic leukemia (B-CLL) harbors deletion in chromosome bands 11q22$\mathrm{q} 23$, and exhibits aggressive phenotype and poor survival [35]. ATM is located at 11q22. Therefore, mutations in the ATM gene in sporadic cases of B-CLL were investigated. Expression of the ATM protein was impaired in $40 \%$ and mutations within ATM were detected in $18 \%$ [36]. A subset of approximately $40 \%$ of patients carrying a del(11q) clone displays inactivating mutations of the second ATM allele and these cases show a poor chemotherapy response, reminiscent of what has been described for TP53defective CLLs [37].

Mantle cell lymphoma (MCL) is genetically characterized by the translocation $\mathrm{t}(11 ; 14)(\mathrm{q} 13 ; \mathrm{q} 32)$ leading to an overexpression of CCND1 (Cyclin-D). Other nonrandom aberrations, especially deletions and, less frequently, translocations, involving bands 11q21-923 have been identified in MCL. Deletion of 11q22-q23 involving ATM was identified to be $46 \%$ in MCL cases $[38,39]$. In addition, deleterious point mutations in the ATM gene were found in $33.3-43 \%$ of MCL cases [40, 41]. There was no difference in TP53 mutation status in the ATM mutant and wild-type groups of MCL. There was no statistically significant difference in the median overall survival of patients with wildtype versus mutated ATM in MCL.

Other than B-CLL and MCL, ATM mutation was infrequently reported in various types of leukemia/lymphoma. Among 120 lymphoid neoplasms, ATM mutations were identified in 9 of 45 diffuse large B cell lymphomas (DLBCLs), 2 of 24 follicular lymphomas, and 1 of 27 adult acute lymphoblastic leukemias [42].

\section{DNA damage response works as a cancerous progression barrier}

Cellular DNA in mammalian cells under constant DNAdamaging stress always carries the risk of mutation. Initially, genetic alternations may take place in genes with potential oncogenic activity, which may then begin progression toward transformation. Oncogenic stimuli enforce cell proliferation, which requires DNA replication. Unscheduled DNA replication enforced by oncogenic stimuli activates a signal for stalled replication, leading to single-strand breaks and subsequently to double-strand breaks on DNA. This DNA damage facilitates activation of DNA damage check point pathways including ATM, which can then lead to cell cycle arrest, apoptosis or cell senescence [43, 44]. However, once DNA damage has occurred in genes playing a crucial role in the DNA damage response itself, the DNA damage check point pathway is compromised and the cells acquire a growth advantage, and can undergo transformation more readily. Such cells, manifesting genomic instability, thus start to proliferate and accumulate additional complex mutations. Tumorigenesis evolves stepwise in this fashion [45, 46] (Fig. 7).

Myelodysplastic syndrome (MDS) is a clonal malignant hematological disorder characterized by ineffective hematopoiesis. Patients with MDS frequently develop overt leukemia after several years of latency. Here, we show that DNA damage check point pathways, such as phosphorylation of ATM, CHEK2, TP53 and H2AFX, are activated in MDS cells, especially in Refractory Anemia with Excess Blasts (RAEB) in phase I and II. However, activation of these DNA damage check point pathways is diminished in overt leukemia (OL). Based on these findings, we propose that DNA damage check point pathways play a crucial role in preventing MDS from progressing to OL. Thus, disruption of DNA damage checkpoint pathways is directly involved in the leukemic transformation of MDS cells [47].

Several studies have directly addressed the relationship between BCR-ABL and molecules involved in DNA repair [48]. They suggested that genomic instability can be caused 
Fig. 7 DNA damage response prevents cancer transformation. Enforced cell proliferation by oncogenic stimuli triggers the cellular DNA damage response (DDR), as a result of aberrations in DNA replication. The DDR then arrests cell proliferation or causes cell death. This might create a selection pressure for suppression of the DDR during carcinogenesis. Therefore, DDR inactivation leads to progression to malignant lesions with genetic instability

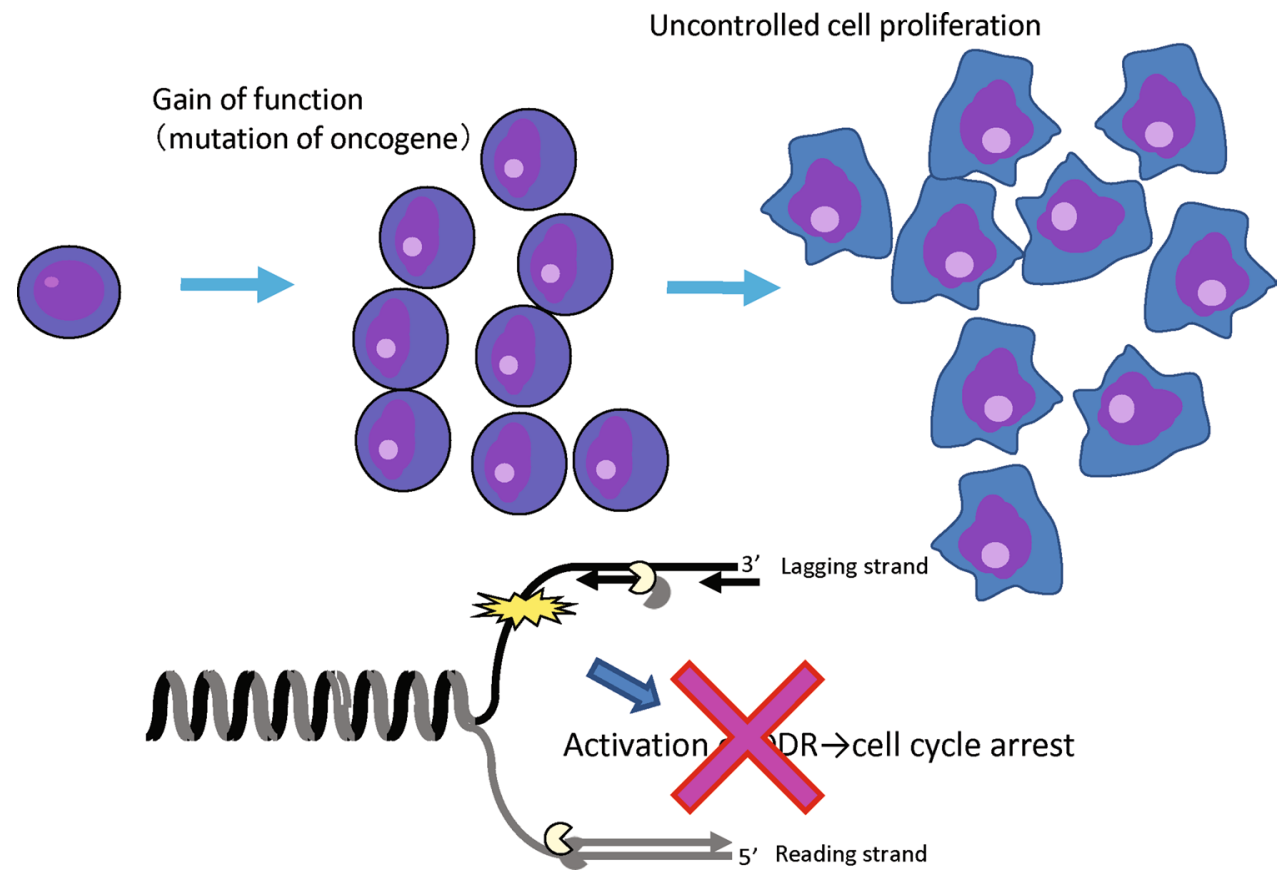

by BCR-ABL. In BCR-ABL-expressing cells, levels of DNA-PK were down-regulated, which may interfere with non-homologous end joining activity [49]. BCR-ABL also interferes with the Fanconi anemia/BRCA pathway [50, 51], or enhances the expression/activity of RAD51, and may induce genomic instability $[52,53]$. Thus, BCR-ABL is a direct inhibitor of genomic stability. One can easily speculate that an increase of genomic instability by BCRABL contributes to blastic crisis of CML. Also, activation of the cell proliferation machinery by BCR-ABL expression may lead to unscheduled DNA replication, to singlestrand breaks and subsequently to double-strand breaks of DNA, and thereby activate the DNA damage response during stalled replication. Therefore, we studied whether loss of this pathway accelerates blast crisis by crossing Atm-knockout mice with BCR-ABL transgenic mice. Interestingly, loss of one of the Atm alleles was shown to be enough for the acceleration of the blast crisis [54]. Under the light of these findings, DNA damage response pathway plays a vital role for determination of susceptibility to blast crisis in CML.

\section{Therapeutic strategy targeting DDR}

Poly(ADP-ribose) polymerase (PARP) exerts various cellbiological effects via poly(ADP) ribosylation of target proteins, many of which are involved in the maintenance of genomic stability, energy metabolism, and cell death [55]. One of the best characterized roles of PARP is the regulation of the DNA damage response pathway [56, 57]. Nucleotide adducts or modifications and DNA cross-linking and alkylation activate the base excision repair (BER) machinery, through which PARP1 is activated by DNA singlestrand breaks (SSBs) to accumulate at break sites, where it recruits DNA repair proteins, including XRCC1 [58, 59]. When PARP function is inhibited, these SSBs are converted to DNA double-strand breaks (DSBs) during DNA replication, which are usually repaired through the homologous recombination repair (HRR) pathway.

BRCA1 and BRCA2 play critical roles in the HRR pathway. Consequently, breast cancers that develop in patients with inherited defects in BRCA1 or BRCA2 are susceptible to synthetic lethality induced by PARP inhibitors [57, 60, 61]. "Synthetic lethality" refers to a situation, such as the use of a PARP inhibitor against HRR-defective tumor cells, in which genetic or pharmaceutical knockout of two proteins, neither of which alone is essential for life, is lethal to the cell. The concept of synthetic lethality is widely applicable to various tumors that lack HRR activity because of genetic alterations.

A few previous reports analyzed the effects of PARP inhibitors on leukemia cells [62, 63]. Recently, Esposito et al. reported that RUNX1-RUNX1T1 and PML-RARA fusion-positive leukemia is extremely sensitive to PARP inhibitors. In contrast, KMT2A translocation-positive leukemia was found to be insensitive to PARP inhibition, an observation that was corroborated by our screening [64]. Furthermore, Faraoni et al. reported that AML-derived primary leukemic blasts exhibit PARP inhibitor sensitivity. In that research, although 1 RUNX1-RUNX1T1 case was identified, the molecular basis was elusive in the other cases. Contrary to the findings reported by Esposito et al., 
Faraoni et al. observed higher sensitivity in KMT2Aassociated leukemic blasts, compared with the RUNX1RUNX1T1 case. Intriguingly, Faraoni and colleagues observed decreased expression levels of BRCA1 and BRCA2 in AML blasts [65]. In addition, myelodysplastic syndrome-derived P39 and AML-derived Mutz- 3 cell lines are sensitive to the PARP inhibitor KU-0058948 [62]. Takagi et al. reported revealed TCF3 (E2A)-HLF-positive leukemia exhibited sensitivity to PARP inhibitor. TCF3-HLF expression suppressed HRR activity, suggesting the induction of synthetic lethality by PARP inhibitor treatment [66].

Acknowledgements I acknowledge mentorship by Shuki Mizutani.

\section{Compliance with ethical standards}

Conflict of interest Author declares no conflict of interest.

\section{References}

1. Caldecott KW. Single-strand break repair and genetic disease. Nat Rev Genet. 2008;9:619-31.

2. Haber JE. DNA recombination: the replication connection. Trends Biochem Sci. 1999;24:271-5.

3. Vilenchik MM, Knudson AG. Endogenous DNA double-strand breaks: production, fidelity of repair, and induction of cancer. Proc Natl Acad Sci USA. 2003;100:12871-6.

4. Uziel T, Lerenthal Y, Moyal L, Andegeko Y, Mittelman L, Shiloh Y. Requirement of the MRN complex for ATM activation by DNA damage. EMBO J. 2003;22:5612-21.

5. Bakkenist CJ, Kastan MB. DNA damage activates ATM through intermolecular autophosphorylation and dimer dissociation. Nature. 2003;421:499-506.

6. Shiloh Y. ATM and related protein kinases: safeguarding genome integrity. Nat Rev Cancer. 2003;3:155-68.

7. Kastan MB, Zhan Q, El-Deiry DW, Carrier F, Jacks T, Walsh WV, Plunkett BS, et al. A mammalian cell cycle checkpoint pathway utilizing p53 and GADD45 is defective in ataxia-telangiectasia. Cell. 1992;71:587-97.

8. Canman CE, Wolff AC, Chen CY, Fornace AJ, Kastan MB. The p53-dependent G1 cell cycle checkpoint pathway and ataxia-telangiectasia. Cancer Res. 1994;54:5054-8.

9. Chehab NH, Malikzay A, Appel M, Halazonetis TD. Chk2/ hCds1 functions as a DNA damage checkpoint in G(1) by stabilizing p53. Genes Dev. 2000;14:278-88.

10. Maya R, Balass M, Kim ST, Shkedy D, Leal JF, Shifman O, Moas M, et al. ATM-dependent phosphorylation of Mdm2 on serine 395: role in p53 activation by DNA damage. Genes Dev. 2001;15:1067-77.

11. Falck J, Mailand N, Syljuasen RG, Bartek J, Lukas J. The ATMChk2-Cdc25A checkpoint pathway guards against radioresistant DNA synthesis. Nature. 2001;410:842-7.

12. Bartek J, Lukas C, Lukas J. Checking on DNA damage in $\mathrm{S}$ phase. Nat Rev Mol Cell Biol. 2004;5:792-804.

13. Qin B, Gao B, Yu J, Yuan J, Lou Z. Ataxia telangiectasiamutated- and Rad3-related protein regulates the DNA damageinduced G2/M checkpoint through the Aurora A cofactor Bora protein. J Biol Chem. 2013;288:16139-44.

14. Menzel T, Nahse-Kumpf V, Kousholt AN, Klein DK, LundAndersen C, Lees M, Johansen JV, et al. A genetic screen identifies BRCA2 and PALB2 as key regulators of G2 checkpoint maintenance. EMBO Rep. 2011;12:705-12.

15. Suijkerbuijk SJ, Kops GJ. Preventing aneuploidy: the contribution of mitotic checkpoint proteins. Biochim Biophys Acta. 2008;1786:24-31.

16. Lew DJ, Burke DJ. The spindle assembly and spindle position checkpoints. Annu Rev Genet. 2003;37:251-82.

17. Yang C, Tang X, Guo X, Niikura Y, Kitagawa K, Cui K, Wong ST, et al. Aurora-B mediated ATM serine 1403 phosphorylation is required for mitotic ATM activation and the spindle checkpoint. Mol Cell. 2011;44:597-608.

18. Perlman S, Becker-Catania S, Gatti RA. Ataxia-telangiectasia: diagnosis and treatment. Semin Pediatr Neurol. 2003;10:173-82.

19. Martin NT, Nahas SA, Tunuguntla R, Fike F, Gatti RA. Assessing 'radiosensitivity' with kinetic profiles of gamma-H2AX, 53BP1 and BRCA1 foci. Radiother Oncol. 2011;101:35-8.

20. Demuth I, Digweed M. The clinical manifestation of a defective response to DNA double-strand breaks as exemplified by Nijmegen breakage syndrome. Oncogene. 2007;26:7792-8.

21. Takagi M, Absalon MJ, McLure KG, Kastan MB. Regulation of p53 translation and induction after DNA damage by ribosomal protein L26 and nucleolin. Cell. 2005;123:49-63.

22. Beckerman R, Prives C. Transcriptional regulation by p53. Cold Spring Harbor Perspect Biol. 2010;2:a000935.

23. Appleby JM, Barber JB, Levine E, Varley JM, Taylor AM, Stankovic T, Heighway $J$, et al. Absence of mutations in the ATM gene in breast cancer patients with severe responses to radiotherapy. Br J Cancer. 1997;76:1546-9.

24. Kleihues P, Schauble B, zur Hausen A, Esteve J, Ohgaki H. Tumors associated with p53 germline mutations: a synopsis of 91 families. Am J Pathol. 1997;150:1-13.

25. Nichols KE, Malkin D, Garber JE, Fraumeni JF Jr, Li FP. Germline p53 mutations predispose to a wide spectrum of early-onset cancers. Cancer Epidemiol Biomarkers Prev. 2001;10:83-7.

26. Holmfeldt L, Wei L, Diaz-Flores E, Walsh M, Zhang J, Ding L, Payne-Turner D, et al. The genomic landscape of hypodiploid acute lymphoblastic leukemia. Nat Genet. 2013;45:242-52.

27. German J, Passarge E. Bloom's syndrome. XII. Report from the Registry for 1987. Clin Genet. 1989;35:57-69.

28. Wu L. Role of the BLM helicase in replication fork management. DNA Repair (Amst). 2007;6:936-44.

29. Mehta PA, Tolar J. Fanconi anemia. In: Pagon RA, Adam MP, Ardinger HH, et al., editors. GeneReviews(R). Seattle: University of Washington; 1993.

30. Mamrak NE, Shimamura A, Howlett NG. Recent discoveries in the molecular pathogenesis of the inherited bone marrow failure syndrome Fanconi anemia. Blood Rev. 2016. doi:10.1016/j. blre.2016.10.002 [Epub ahead of print].

31. Lynch HT, de la Chapelle A. Hereditary colorectal cancer. N Engl J Med. 2003;348:919-32.

32. Ripperger T, Schlegelberger B. Acute lymphoblastic leukemia and lymphoma in the context of constitutional mismatch repair deficiency syndrome. Eur J Med Genet. 2016;59:133-42.

33. Lavoine N, Colas C, Muleris M, Bodo S, Duval A, Entz-Werle $\mathrm{N}$, Coulet $\mathrm{F}$, et al. Constitutional mismatch repair deficiency syndrome: clinical description in a French cohort. J Med Genet. 2015;52:770-8.

34. Vorechovsky I, Luo L, Dyer MJ, Catovsky D, Amlot PL, Yaxley JC, Foroni L, et al. Clustering of missense mutations in the ataxia-telangiectasia gene in a sporadic T-cell leukaemia. Nat Genet. 1997;17:96-9.

35. Dohner H, Stilgenbauer S, James MR, Benner A, Weilguni T, Bentz M, Fischer K, et al. $11 \mathrm{q}$ deletions identify a new subset of B-cell chronic lymphocytic leukemia characterized by extensive nodal involvement and inferior prognosis. Blood. 1997;89:2516-22. 
36. Stankovic T, Weber P, Stewart G, Bedenham T, Murray J, Byrd PJ, Moss PA, et al. Inactivation of ataxia telangiectasia mutated gene in B-cell chronic lymphocytic leukaemia. Lancet. 1999;353:26-9.

37. Austen B, Skowronska A, Baker C, Powell JE, Gardiner A, Oscier D, Majid A, et al. Mutation status of the residual ATM allele is an important determinant of the cellular response to chemotherapy and survival in patients with chronic lymphocytic leukemia containing an 11q deletion. J Clin Oncol. 2007;25:5448-57.

38. Stilgenbauer S, Liebisch P, James MR, Schroder M, Schlegelberger B, Fischer K, Bentz M, et al. Molecular cytogenetic delineation of a novel critical genomic region in chromosome bands 11q22.3-923.1 in lymphoproliferative disorders. Proc Natl Acad Sci USA. 1996;93:11837-41.

39. Stilgenbauer S, Schaffner C, Winkler D, Ott G, Leupolt E, Bentz $\mathrm{M}$, Moller P, et al. The ATM gene in the pathogenesis of mantlecell lymphoma. Ann Oncol. 2000;11(Suppl 1):127-30.

40. Fang NY, Greiner TC, Weisenburger DD, Chan WC, Vose JM, Smith LM, Armitage JO, et al. Oligonucleotide microarrays demonstrate the highest frequency of ATM mutations in the mantle cell subtype of lymphoma. Proc Natl Acad Sci USA. 2003;100:5372-7.

41. Greiner TC, Dasgupta C, Ho VV, Weisenburger DD, Smith LM, Lynch JC, Vose JM, et al. Mutation and genomic deletion status of ataxia telangiectasia mutated (ATM) and p53 confer specific gene expression profiles in mantle cell lymphoma. Proc Natl Acad Sci USA. 2006;103:2352-7.

42. Gronbaek K, Worm J, Ralfkiaer E, Ahrenkiel V, Hokland P, Guldberg P. ATM mutations are associated with inactivation of the ARF-TP53 tumor suppressor pathway in diffuse large B-cell lymphoma. Blood. 2002;100:1430-7.

43. Bakkenist CJ, Kastan MB. Initiating cellular stress responses. Cell. 2004;118:9-17.

44. Bartkova J, Rezaei N, Liontos M, Karakaidos P, Kletsas D, Issaeva $\mathrm{N}$, Vassiliou $\mathrm{LV}$, et al. Oncogene-induced senescence is part of the tumorigenesis barrier imposed by DNA damage checkpoints. Nature. 2006;444:633-7.

45. Bartkova J, Horejsi Z, Koed K, Kramer A, Tort F, Zieger K, Guldberg $\mathrm{P}$, et al. DNA damage response as a candidate anti-cancer barrier in early human tumorigenesis. Nature. 2005;434:864-70.

46. Gorgoulis VG, Vassiliou LV, Karakaidos P, Zacharatos P, Kotsinas A, Liloglou T, Venere M, et al. Activation of the DNA damage checkpoint and genomic instability in human precancerous lesions. Nature. 2005;434:907-13.

47. Horibe S, Takagi M, Unno J, Nagasawa M, Morio T, Arai $\mathrm{A}$, Miura $\mathrm{O}$, et al. DNA damage check points prevent leukemic transformation in myelodysplastic syndrome. Leukemia. 2007;21:2195-8.

48. Skorski T. Genomic instability: the cause and effect of BCR/ ABL tyrosine kinase. Curr Hematol Malig Rep. 2007;2:69-74.

49. Poplawski T, Blasiak J. BCR/ABL downregulates DNA-PK(CS)dependent and upregulates backup non-homologous end joining in leukemic cells. Mol Biol Rep. 2010;37:2309-15.

50. Valeri A, Alonso-Ferrero ME, Rio P, Pujol MR, Casado JA, Perez $\mathrm{L}$, Jacome A, et al. Bcr/Abl interferes with the Fanconi anemia/ BRCA pathway: implications in the chromosomal instability of chronic myeloid leukemia cells. PLoS One. 2010;5:e15525.

51. Deutsch E, Jarrousse S, Buet D, Dugray A, Bonnet ML, Vozenin-Brotons MC, Guilhot F, et al. Down-regulation of
BRCA1 in BCR-ABL-expressing hematopoietic cells. Blood. 2003;101:4583-8.

52. Slupianek A, Jozwiakowski SK, Gurdek E, Skorski T. BCR/ABL kinase interacts with and phosphorylates the RAD51 paralog, RAD51B. Leukemia. 2009;23:2308-10.

53. Slupianek A, Schmutte C, Tombline G, Nieborowska-Skorska M, Hoser G, Nowicki MO, Pierce AJ, et al. BCR/ABL regulates mammalian RecA homologs, resulting in drug resistance. Mol Cell. 2001;8:795-806.

54. Takagi M, Sato M, Piao J, Miyamoto S, Isoda T, Kitagawa M, Honda $\mathrm{H}$, et al. ATM-dependent DNA damage-response pathway as a determinant in chronic myelogenous leukemia. DNA Repair (Amst). 2013;12:500-7.

55. Miwa M, Masutani M. PolyADP-ribosylation and cancer. Cancer Sci. 2007;98:1528-35.

56. Satoh MS, Lindahl T. Role of poly(ADP-ribose) formation in DNA repair. Nature. 1992;356:356-8.

57. Helleday T, Petermann E, Lundin C, Hodgson B, Sharma RA. DNA repair pathways as targets for cancer therapy. Nat Rev Cancer. 2008;8:193-204.

58. Audebert M, Salles B, Calsou P. Involvement of poly(ADPribose) polymerase-1 and XRCC1/DNA ligase III in an alternative route for DNA double-strand breaks rejoining. J Biol Chem. 2004;279:55117-26.

59. El-Khamisy SF, Masutani M, Suzuki H, Caldecott KW. A requirement for PARP-1 for the assembly or stability of XRCC1 nuclear foci at sites of oxidative DNA damage. Nucleic Acids Res. 2003;31:5526-33.

60. Bryant HE, Schultz N, Thomas HD, Parker KM, Flower D, Lopez E, Kyle S, et al. Specific killing of BRCA2-deficient tumours with inhibitors of poly(ADP-ribose) polymerase. Nature. 2005;434:913-7.

61. Farmer H, McCabe N, Lord CJ, Tutt AN, Johnson DA, Richardson TB, Santarosa M, et al. Targeting the DNA repair defect in BRCA mutant cells as a therapeutic strategy. Nature. 2005;434:917-21.

62. Gaymes TJ, Shall S, MacPherson LJ, Twine NA, Lea NC, Farzaneh F, Mufti GJ. Inhibitors of poly ADP-ribose polymerase (PARP) induce apoptosis of myeloid leukemic cells: potential for therapy of myeloid leukemia and myelodysplastic syndromes. Haematologica. 2009;94:638-46.

63. Horton TM, Jenkins G, Pati D, Zhang L, Dolan ME, RibesZamora A, Bertuch AA, et al. Poly(ADP-ribose) polymerase inhibitor ABT-888 potentiates the cytotoxic activity of temozolomide in leukemia cells: influence of mismatch repair status and O6-methylguanine-DNA methyltransferase activity. Mol Cancer Ther. 2009;8:2232-42.

64. Esposito MT, Zhao L, Fung TK, Rane JK, Wilson A, Martin N, Gil J, et al. Synthetic lethal targeting of oncogenic transcription factors in acute leukemia by PARP inhibitors. Nat Med. 2015;21:1481-90.

65. Faraoni I, Compagnone M, Lavorgna S, Angelini DF, Cencioni MT, Piras E, Panetta P, et al. BRCA1, PARP1 and gammaH2AX in acute myeloid leukemia: role as biomarkers of response to the PARP inhibitor olaparib. Biochim Biophys Acta. 2015;1852:462-72.

66. Piao J, Takai S, Kamiya T, Inukai T, Sugita K, Ohyashiki K, Delia D, et al. Poly (ADP-ribose) polymerase inhibitors selectively induce cytotoxicity in TCF3-HLF-positive leukemic cells. Cancer Lett. 2017;386:131-40. 